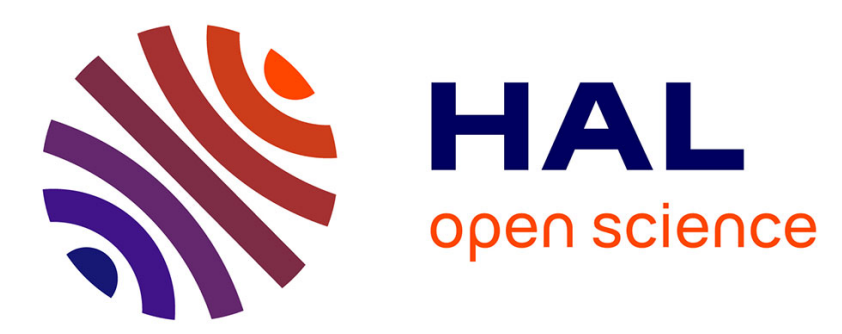

\title{
Polymorphisme du 4-cyano-4'-nonylbiphényl : étude par diffusion Raman de la région des vibrations de valence $\nu$ CH de la chaîne aliphatique
}

\author{
M. Jaffrain, G. Lacrampe, G. Martin
}

\section{- To cite this version:}

M. Jaffrain, G. Lacrampe, G. Martin. Polymorphisme du 4-cyano-4'-nonylbiphényl: étude par diffusion Raman de la région des vibrations de valence $\nu \mathrm{CH}$ de la chaîne aliphatique. Journal de Physique Lettres, 1984, 45 (22), pp.1103-1110. 10.1051/jphyslet:0198400450220110300 • jpa-00232456

\section{HAL Id: jpa-00232456 https://hal.science/jpa-00232456}

Submitted on 1 Jan 1984

HAL is a multi-disciplinary open access archive for the deposit and dissemination of scientific research documents, whether they are published or not. The documents may come from teaching and research institutions in France or abroad, or from public or private research centers.
L'archive ouverte pluridisciplinaire HAL, est destinée au dépôt et à la diffusion de documents scientifiques de niveau recherche, publiés ou non, émanant des établissements d'enseignement et de recherche français ou étrangers, des laboratoires publics ou privés. 
Classification

Physics Abstracts

$61.30 \mathrm{E}-78.30$

\title{
Polymorphisme du 4-cyano-4' -nonylbiphényl : étude par diffusion Raman de la région des vibrations de valence $\nu_{C H}$ de la châine aliphatique
}

\author{
M. Jaffrain, G. Lacrampe et G. Martin \\ Laboratoire de Physique Moléculaire et Biologique, Université René Descartes, \\ 45, rue des Saints-Pères, 75270 Paris Cedex 06, France
}

(Reçu le 27 juillet 1984, accepté le 27 septembre 1984)

\begin{abstract}
Résumé. - Nous présentons les résultats expérimentaux préliminaires d'une étude du polymorphisme des cristaux liquides par l'intermédiaire de la région des vibrations de valence $v_{C H}$ de la chaîne aliphatique. Utilisant le fait que les fréquences et les intensités de ces modes sont sensibles à la conformation des chaînes et aux interactions latérales entre celles-ci, nous proposons une structure possible des deux formes solides conduisant à l'interprétation des formes smectiques partiellement bicouches des cyanobiphényls. A notre connaissance, une telle étude n'avait pas encore été faite pour les cristaux liquides thermotropes.
\end{abstract}

\begin{abstract}
This paper presents preliminary experimental results of a vibrational spectroscopic study in the C-H stretching region of the aliphatic chain of cyanobiphenyls. The now well-established use of Raman spectroscopy to examine the structure and conformation of aliphatic chains permits' us to propose possible structures for the two solid phases of 4-cyano-4'-nonylbiphenyl which lead to the partial bilayer structure of the smectic mesophase. To our knowledge, such a study has not been previously made for thermotropic liquid crystals.
\end{abstract}

\section{Introduction.}

Des expériences récentes, portant sur la structure de la bicouche lipidique des biomembranes ont montré l'intérêt que présente l'étude de la région des vibrations de valence des liaisons méthylène pour obtenir des informations sur les interactions intra et intermoléculaires dans les chaînes aliphatiques [1-4].

Il est apparu que les variations des fréquences et de l'intensité relative des raies de vibration Raman et d'absorption infrarouge pouvaient être corrélées à la conformation des chaînes, à l'environnement et à la dynamique des chaînes aliphatiques [5-6]. Ces observations ont redonné une impulsion à l'interprétation du spectre vibrationnel de cette région : la complexité de celui-ci résulte dans une large mesure d'interactions de résonance de Fermi des modes de vibration de valence avec les harmoniques des modes de déformation des groupements méthylène, ces modes étant eux-mêmes influencés par le couplage interchaîne [7-13].

Nous nous sommes proposés d'appliquer ces particularités du spectre Raman de la région $v_{\mathrm{CH}}$ aliphatique au cas des cristaux liquides thermotropes pour lesquels la conformation et l'arran- 
gement relatif des chaînes latérales joue un rôle important, tant pour l'obtention des phases mésomorphes que pour la structure des phases solides polymorphes.

Le groupe des cyanobiphényls nous a paru particulièrement intéressant dans la mesure où divers modèles d'arrangement relatif des molécules mésogènes ont été proposés pour rendre compte de la structure bicouche ou partiellement bicouche des phases smectiques [14-18].

Nous avons surtout examiné les phases solides; des expériences antérieures ont en effet montré que le spectre des phases mésomorphes pouvait être relié à celui des formes polymorphes solides [19].

Nous présentons ici des résultats préliminaires sur le p-nonyl-p'-cyanobiphényl $(9 \mathrm{CB})$ pour lequel, en plus de la forme solide stable donnant lieu à la forme smectique A vers $42^{\circ} \mathrm{C}$, on peut observer dans certains cas, une forme solide métastable, dont le point de transition solide-smectique A se situe vers $29^{\circ} \mathrm{C}$ [20-21]. Nous n'avons pas étudié la phase nématique dont le domaine d'existence n'est que de $1,5^{\circ} \mathrm{C}$ à pression ordinaire; la transition vers le liquide isotrope a été observée à $49^{\circ} \mathrm{C}$.

\section{Conditions expérimentales.}

Les spectres de diffusion Raman ont été enregistrés avec un spectromètre Jobin-Yvon, type Ramanor HG 2S, après avoir été excités par la raie verte $(\lambda=5145 \AA)$ d'un laser à argon ionisé SPECTRA PHYSICS. La puissance maximum utilisée a été de $300 \mathrm{~mW}$ pour l'étude de la forme stable de l'échantillon; nous n'avons pas pu dépasser $200 \mathrm{~mW}$ pour la forme métastable.

Le p-nonyl-p'-cyanobiphényl (9.CB) est un produit MERCK, utilisé sans purification ultérieure. L'échantillon est enfermé dans un tube capillaire de $1 \mathrm{~mm}$ de diamètre. Celui-ci est disposé dans l'enceinte d'un minicryostat CODERG, type Cryocirc, à circulation d'azote, muni d'une régulation de température permettant une stabilité en température de l'ordre du demi-degré.

Le $9 \mathrm{CB}$ est solide à température ordinaire et les tubes capillaires sont remplis après avoir porté l'échantillon à une température supérieure à la température de transition nématique-liquide isotrope. Ramené à la température ordinaire, l'échantillon cristallise normalement dans la forme stable. La forme métastable n'a pu être obtenue qu'en refroidissant très lentement l'échantillon à partir de la température à laquelle le tube est rempli, jusqu'à la température ordinaire. Il peut alors être maintenu à cette température pendant plusieurs jours, être refroidi à la température de l'azote liquide sans se transformer en forme stable, à condition d'éviter un choc thermique et de ne pas l'éclairer sous une trop forte intensité laser. Le refroidissement de la phase smectique nous a toujours donné la forme stable.

\section{Résultats expérimentaux et discussion.}

Les figures 1 à 6 représentent les spectres de la chaîne aliphatique (vibrations de valence des groupes methyl et méthylène entre 2750 et $3000 \mathrm{~cm}^{-1}$, vibrations de déformation des groupes méthylène vers $1450 \mathrm{~cm}^{-1}$ ) pour les deux formes solides observées (Figs. 1 à 4), la phase smectique (Fig. 5) et le liquide isotrope (Fig. 6). Les vibrations de valence $v_{\mathrm{CH}}$ du cour aromatique se situent à fréquence plus élevée ( 3000 à $3100 \mathrm{~cm}^{-1}$ ) et n'interfèrent pas avec ceux de la chaîne aliphatique.

Les attributions des fréquences des groupes méthyl et méthylène ont été discutées par Snyder et al. [7] et nous avons adopté ses notations : les deux vibrations symétrique $\left(\mathrm{d}^{+}\right)$et antisymétrique $\left(\mathrm{d}^{-}\right)$du groupe méthylène sont respectivement observées aux environs de 2850 et $2880 \mathrm{~cm}^{-1}$ dans le spectre Raman de la chaîne polyméthylène. Les 3 modes de vibration du groupe méthyl : la vibration totalement symétrique $\left(\mathrm{r}^{+}\right)$se situe à $2871 \mathrm{~cm}^{-1}$, la vibration asymétrique $\left(\mathrm{r}_{\mathrm{a}}^{-}\right)$ dans le plan du squelette à $2964 \mathrm{~cm}^{-1}$, la vibration asymétrique hors plan du squelette $\left(\mathrm{r}_{\mathrm{b}}^{-}\right)$à $2952 \mathrm{~cm}^{-1}$. La vibration de déformation $\delta$ de l'angle $\mathrm{HCH}$ est observée à $1443 \mathrm{~cm}^{-1}$ dans le spectre Raman de la chaîne polyméthylène.

Dans la chaîne finie (alcanes) ou infinie (polyméthylène) le couplage entre les groupes méthy- 
lène peut être décrit en termes du déphasage entre les vibrations de groupes $\mathrm{CH}_{2}$ voisins et conduit à une dispersion des fréquences en fonction de l'angle de déphasage.

Cette dispersion est importante pour les modes $\delta_{\mathbf{C H}_{2}}$; il en résulte une forte dispersion des combinaisons binaires de ces modes qui, selon leur type de symétrie, peuvent interagir avec les modes de vibration de valence, avec pour conséquence un élargissement du spectre dans la région 2750 à $3000 \mathrm{~cm}^{-1}$. La structure du massif dépend donc des différents facteurs influençant la dispersion de ces modes et leur type de symétrie et par conséquent de la conformation de la chaîne et des interactions latérales.

3. 1 FORMES SOLIDES. - Les spectres des deux formes se différencient nettement l'un de l'autre et ont une évolution en température très différente. Dans les deux cas, on observe un spectre fin et bien résolu à basse température (Figs. 1 et 3); cependant, alors que celui de la forme stable n'évolue que légèrement en fonction de la température (Figs. 1 et 2), jusqu'à la transition solidesmectique, il n'en est pas de même pour la forme métastable dont le spectre s'élargit considérablement entre $80 \mathrm{~K}$ et $295 \mathrm{~K}$ (Figs. 3 et 4).

Dans la forme stable, le spectre est dominé, à $80 \mathrm{~K}$ (Fig. 1), par une raie étroite à $2879 \mathrm{~cm}^{-1}$ attribuée à la vibration antisymétrique $\mathrm{d}^{-}$du groupe $\mathrm{CH}_{2}$; les raies observées vers $2850 \mathrm{~cm}^{-1}$ sont associées à la vibration symétrique $\mathrm{d}^{+}$; il en est de même pour l'ensemble du massif large s'étendant jusqu'à $2950 \mathrm{~cm}^{-1}$ par suite des résonances de Fermi avec les harmoniques de $\delta_{\mathrm{CH}_{2}}$ : on note en particulier des pics à $2890,2920,2930 \mathrm{~cm}^{-1}$ [8]; il en résulte que l'intensité intégrée $\mathrm{du}$ fondamental $\mathrm{d}^{+}$est supérieure à celle du mode $\mathrm{d}^{-}$. Les raies du groupe méthyle sont généralement observées à $2871 \mathrm{~cm}^{-1}\left(\mathrm{r}^{+}\right), 2951 \mathrm{~cm}^{-1}\left(\mathrm{r}_{\mathrm{b}}^{-}\right)$et $2962 \mathrm{~cm}^{-1}\left(\mathrm{r}_{\mathrm{a}}^{-}\right)$. Le splitting de $11 \mathrm{~cm}^{-1}$ entre les deux modes asymétriques $\mathrm{r}_{\mathrm{a}}^{-}$et $\mathrm{r}_{\mathrm{b}}^{-}$résulte du couplage intramoléculaire du groupe $\mathrm{CH}_{3}$ avec le reste de la chaîne quand le groupe méthyle ne tourne pas [10]. Entre $80 \mathrm{~K}$ et la température ordinaire, le déplacement en fréquence est très faible, et la largeur à mi-hauteur de la raie principale sensiblement constante $\left(7 \mathrm{~cm}^{-1}\right.$ à $80 \mathrm{~K}, 9,5 \mathrm{~cm}^{-1}$ à $\left.300 \mathrm{~K}\right)$. On constate également que, mise à part une légère diminution de la résolution, le spectre de la région $\delta_{\mathrm{CH}_{2}}$ est le même à basse et haute température.

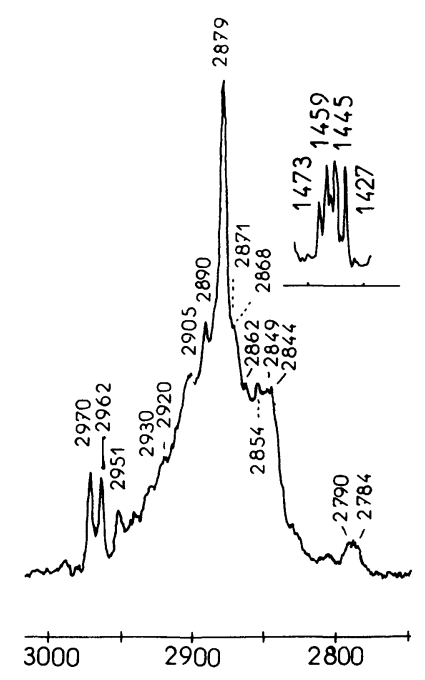

Fig. 1. - Spectre Raman de la chaîne aliphatique $\left(v_{\mathrm{CH}}, \delta_{\mathrm{CH}}\right)$ du p-nonyl-p'-cyanobiphényl. Solide stable à $T=80 \mathrm{~K}$.

[Raman vibrational spectra of the aliphatic part $\left(v_{\mathrm{CH}}, \delta_{\mathrm{CH}}\right)$ of p-nonyl-p'-cyanobiphenyl. Stable form at $T=80 \mathrm{~K}$. 


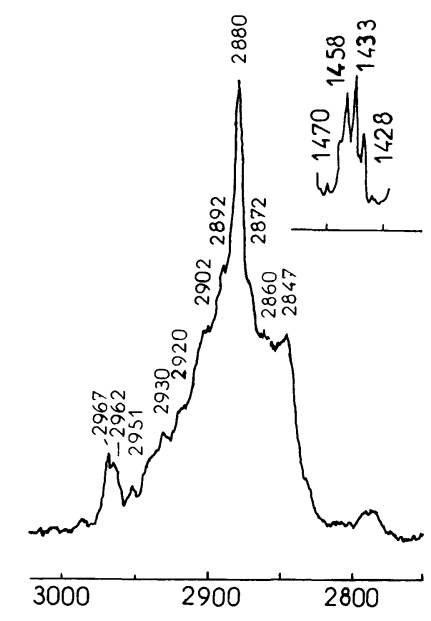

Fig. 2. - Spectre Raman de la chaîne aliphatique $\left(v_{\mathrm{CH}}, \delta_{\mathrm{CH}}\right)$ du p-nonyl-p'-cyanobiphényl. Solide stable à $T=300 \mathrm{~K}$.

[Raman vibrational spectra of the aliphatic part $\left(v_{\mathrm{CH}}, \delta_{\mathrm{CH}}\right)$ of p-nonyl-p'-cyanobiphenyl. Stable form at $T=300 \mathrm{~K}$.]

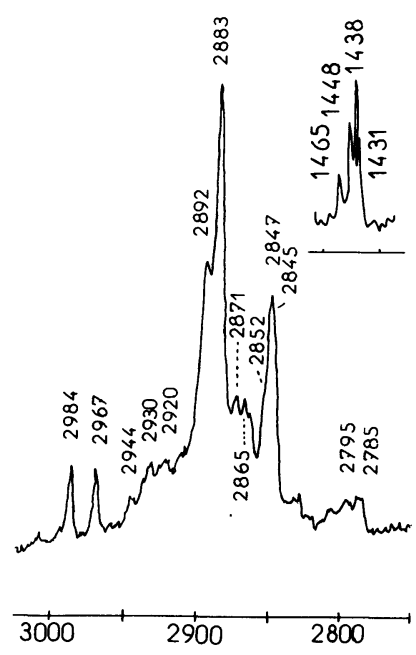

Fig. 3. - Spectre Raman de la chaîne aliphatique $\left(v_{\mathrm{CH}}, \delta_{\mathrm{CH}}\right)$ du p-nonyl-p'-cyanobiphényl. Solide métastable à $T=80 \mathrm{~K}$.

[Raman vibrational spectra of the aliphatic part $\left(v_{\mathrm{CH}}, \delta_{\mathrm{CH}}\right)$ of p-nonyl-p'-cyanobiphenyl. Metastable form at $T=80 \mathrm{~K}$.]

L'aspect général du spectre entre 2800 et $3000 \mathrm{~cm}^{-1}$ conduirait à supposer, en se basant sur les propositions de Snyder [7], une structure triclinique pour cette forme.

Cette structure correspond à un empilement très compact de chaînes, donc à une faible mobilité de celles-ci, en accord avec la faible évolution du spectre en température. 
Cependant, le réseau triclinique ne comprend qu’une molécule par maille; il en résulterait une structure ferroélectrique à cause de la présence du groupe polaire.

Il est par contre possible d'envisager une structure dans laquelle les molécules seraient disposées en couches, les chaînes étant parallèles les unes aux autres. Un empilement compact pourrait être réalisé avec une couche bimoléculaire du type proposé par D. Guillon et A. Skoulios [22] dans laquelle les molécules s'associeraient par dimères antiparallèles tête à tête; dans ce cas l'encombrement latéral des chaînes et des coeurs aromatiques sont très voisins $\left(\simeq 20 \AA^{2}\right)$.

Dans la forme métastable, on observe, à $80 \mathrm{~K}$ (Fig. 3) deux bandes fines (environ $7 \mathrm{~cm}^{-1}$ à mi-hauteur), une raie intense à $2883 \mathrm{~cm}^{-1}\left(\mathrm{~d}^{-}\right)$et une raie de plus faible intensité à $2845-$ $47 \mathrm{~cm}^{-1}\left(\mathrm{~d}^{+}\right)$. La raie à $2892 \mathrm{~cm}^{-1}$ sur le flanc de la raie à $2880 \mathrm{~cm}^{-1}$ est plus intense que dans la forme stable. Une moins grande dispersion des modes $\delta_{\mathrm{CH}_{2}}$ se traduit, dans la région des vibrations de valence $v_{\mathrm{CH}}$ par un spectre globalement mieux résolu. Contrairement à ce qui a été observé dans la forme stable, l'évolution en température est ici importante. Les deux raies principales s'élargissent $\left(\simeq 14 \mathrm{~cm}^{-1}\right.$ de largeur à mi-hauteur) et on note un accroissement notable de la raie à $2898 \mathrm{~cm}^{-1}$ qui n'est plus qu'un épaulement sur le flanc haute fréquence de la raie à $2884 \mathrm{~cm}^{-1}$, et entraîne une augmentation supplémentaire de la largeur apparente du massif. Simultanément le spectre des déformations $\delta_{\mathrm{CH}_{2}}$ se simplifie. Avec ses deux raies à 2880 et $2850 \mathrm{~cm}^{-1}$, l'allure générale du massif rappelle les spectres observés pour des alcanes cristallisés sous forme orthorhombique ou monoclinique [7]. Cependant le rapport des hauteurs de raies, la largeur de la raie à $2850 \mathrm{~cm}^{-1}$ (égale à celle de la raie à $2880 \mathrm{~cm}^{-1}$, alors qu'elle est beaucoup plus large dans les formes orthorhombiques et monocliniques) font que le spectre à $80 \mathrm{~K}$ est beaucoup plus proche de ceux qui sont observés dans des chaînes quasi isolées (solutions isotopiques [7] clathrates d'urée [23]). A haute température, l'augmentation importante de la largeur apparente de la raie $2884-2898 \mathrm{~cm}^{-1}$ met en évidence une mobilité plus grande des chaînes, avec peut-être l'apparition de formes gauches. En effet, la raie d $\mathrm{d}^{-}$se déplace de $2884 \mathrm{~cm}^{-1}$ à $2890 \mathrm{~cm}^{-1}$ dans le polyéthylène lorsque celui-ci passe du cristal au liquide [12]. Notons cependant que dans les clathrates, la largeur de cette raie augmente uniquement par suite des possibilités accrues de rotation de la chaîne all-trans autour de son axe [23]. Il apparaît donc que, dans la

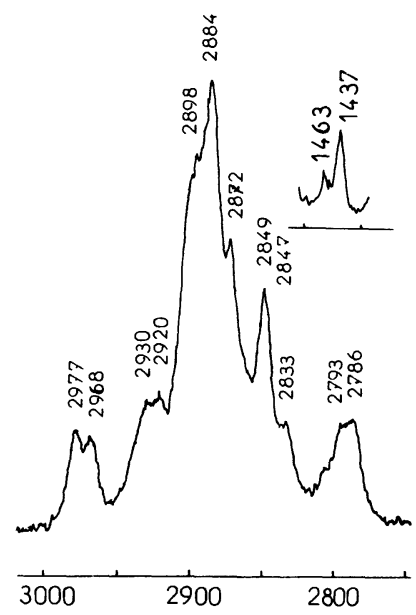

Fig. 4. - Spectre Raman de la chaîne aliphatique $\left(v_{\mathrm{CH}}, \delta_{\mathrm{CH}}\right)$ du p-nonyl-p'-cyanobiphényl. Solide métastable à $T=295 \mathrm{~K}$.

[Raman vibrational spectra of the aliphatic part $\left(v_{\mathrm{CH}}, \delta_{\mathrm{CH}}\right)$ of p-nonyl-p'-cyanobiphenyl. Metastable form at $T=295 \mathrm{~K}$.] 
phase métastable, les interactions latérales entre les chaînes sont plus faibles que dans la forme stable. Ceci pourrait être dû, soit à une disposition relative des molécules dans laquelle il y aurait recouvrement partiel des noyaux biphényls comme dans la phase haute température du 4-cyano4'-n-propylbiphényl [24], soit à une structure en couche monomoléculaire avec juxtaposition antiparallèle côte à côte des molécules, comme l'ont proposé Guillon et Skoulios pour les phases smectiques [22]. Cette dernière structure permettrait une "fusion " facile des chaînes et expliquerait l'abaissement du point de transition solide-smectique par rapport à celui de la forme stable.

3.2 Formes SMECTIQUE ET ISOTROPE. - Dans les deux phases, le massif des vibrations de valence $v_{\mathrm{CH}}$ est très large(Figs. 5 et 6). Celui de la phase liquide présente les caractéristiques habituellement observées dans les alcanes liquides avec prédominance de la raie à $2850 \mathrm{~cm}^{-1}$. Dans le spectre de la phase smectique, l'intensité apparente de cette raie est encore inférieure à celle de l'ensemble du reste du massif. Bien que les bases théoriques indispensables à la compréhension complète de ces spectres ne soient pas encore disponibles, Zerbi et al. $[9,10]$ ont montré que le couplage intramoléculaire des modes $v_{\mathrm{CH}}$ varie quand on passe des structures trans aux structures gauches par suite du changement des fréquences de vibration de valence et de la modification de la courbe de dispersion des modes de déformation. En particulier, ils ont montré que l'intensité de la raie à $2935 \mathrm{~cm}^{-1}$ augmente en présence de conformations gauches et ont proposé de prendre le rapport des intensités à 2850 et $2935 \mathrm{~cm}^{-1}$ comme indicateur de la proportion de liaisons gauches dans le milieu. Appliquée aux formes smectique et liquide, cette proposition indique un pourcentage de formes gauches plus important dans la phase smectique que dans la phase liquide. Une observation analogue a été faite par Wunder et al. [9] qui ont trouvé un pourcentage de formes gauches pour des solutions d'hexadécane dans le chloroforme supérieur à celui qui existe dans l'hexadécane liquide. Le maintien, en phase smectique, d'une certaine interaction latérale entre les chaînes se déduit du rapport des hauteurs de raies à 2890 et $2850 \mathrm{~cm}^{-1}$ : en phase isotrope, ce rapport vaut 0,8 et correspond à la valeur résiduelle en phase liquide d'après Gaber et Petitcolas [5]. En phase smectique, il est encore supérieur à l'unité $(\simeq 1,1)$, tout en étant très

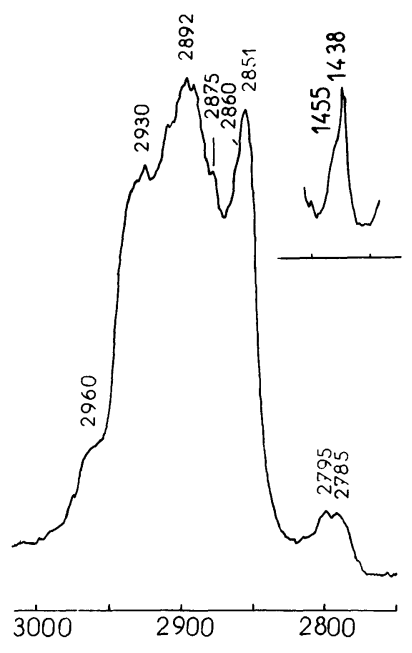

Fig. 5. - Spectre Raman de la chaîne aliphatique $\left(v_{\mathrm{CH}}, \delta_{\mathrm{CH}}\right)$ du p-nonyl-p'-cyanobiphényl. Phase smectique à $T=318 \mathrm{~K}$.

[Raman vibrational spectra of the aliphatic part $\left(v_{\mathrm{CH}}, \delta_{\mathrm{CH}}\right)$ of p-nonyl-p'-cyanobiphenyl. Smectic phase at $T=318 \mathrm{~K}$.] 


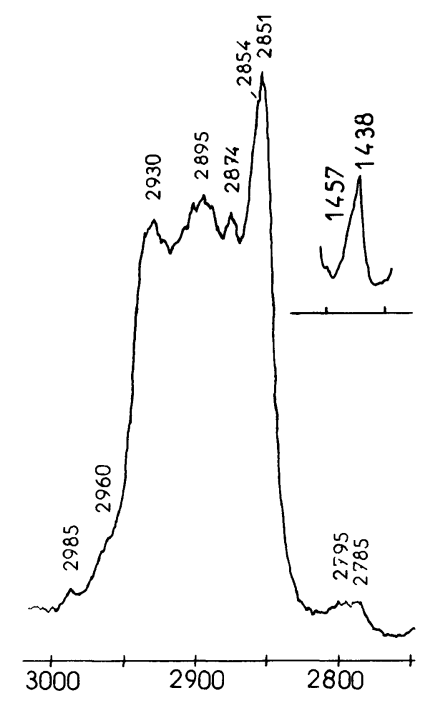

Fig. 6. - Spectre Raman de la chaîne aliphatique $\left(v_{\mathrm{CH}}, \delta_{\mathrm{CH}}\right)$ du p-nonyl-p'-cyanobiphényl. Phase isotrope à $T=325 \mathrm{~K}$.

[Raman vibrational spectra of the aliphatic part $\left(v_{\mathrm{CH}}, \delta_{\mathrm{CH}}\right)$ of p-nonyl-p'-cyanobiphenyl. Isotropic liquid at $T=325 \mathrm{~K}$.]

inférieur à sa valeur en phase solide ordonnée ( $>2)$; il traduit une diminution des interactions latérales et la présence de rotamères. Sa valeur, très proche de celle de la phase isotrope et très éloignée de celle de la phase solide, montre que les chaînes aliphatiques sont fortement désorganisées dans la phase mésomorphe.

\section{Conclusion.}

Une première analyse du spectre de vibrations de la région des vibrations de valence $v_{\mathrm{CH}}$ des chaînes aliphatiques nous a permis de proposer quelques hypothèses concernant la structure et la mobilité des chaînes dans les phases solides, smectique et isotrope du p-nonyl-p'-cyanobiphényl. La forme solide stable pourrait être triclinique avec un dimère par maille. L'empilement des chaînes dans la forme métastable est beaucoup moins compact et permet une mobilité accrue des chaînes, donc des molécules quand on approche de la transition solide-smectique. Une structure en couches moléculaires pourrait en rendre compte. Nous avons également noté que la raie de vibration $v_{\mathrm{C} \equiv \mathrm{N}}$ dépend de la nature de la phase observée : dans la forme stable, elle est simple et fine et se situe à $2223 \mathrm{~cm}^{-1}$ à $80 \mathrm{~K}, 2222 \mathrm{~cm}^{-1}$ à $300 \mathrm{~K}$. Elle présente deux composantes dans la forme métastable, fines et bien résolues (2 229 et $2232 \mathrm{~cm}^{-1}$ à $\left.80 \mathrm{~K}\right)$, plus larges à $295 \mathrm{~K}\left(2230 \mathrm{~cm}^{-1}\right.$ et un épaulement à $\left.2229 \mathrm{~cm}^{-1}\right)$. Gray et Mosley [25] avaient également observé deux raies pour cette vibration dans le p-cyano-p'-pentylbiphényl solide, mais à 2230 et $2223 \mathrm{~cm}^{-1}$, ce qui laisserait supposer la présence d'un mélange de deux formes. En phases smectique et liquide isotrope, la vibration $v_{\mathrm{C} \equiv N}$ donne lieu à une raie unique, plus large et située à $2228 \mathrm{~cm}^{-1}$, fréquence intermédiaire entre les deux précédentes (Fig. 7). Cette observation va dans le sens d'une interprétation de la phase smectique résultant d'un mélange des deux structures solides à basse température et comprenant des groupes de molécules dimères ainsi que des monomères. L'ensemble des spectres des différentes phases sera discuté dans un autre article. 


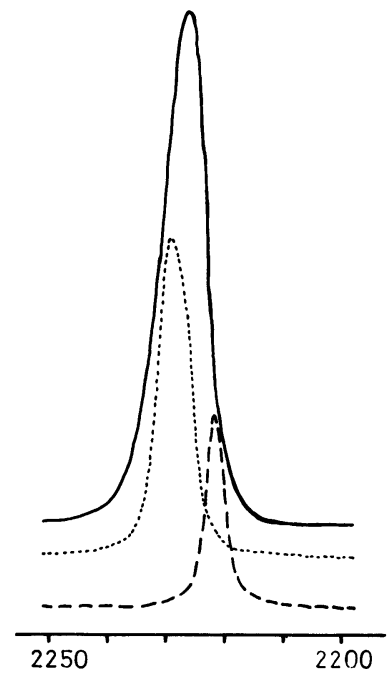

Fig. 7. - Raie de vibration $v_{\mathrm{C} \equiv \mathrm{N}}$ (intensités relatives non normalisées) $(\longrightarrow$ phase smectique à $T=318 \mathrm{~K}$, $(--)$ solide métastable à $T=295 \mathrm{~K},(-\longrightarrow)$ solide stable à $T=300 \mathrm{~K}$.

[Stretching vibration of the $\mathrm{C} \equiv \mathrm{N}$ group (relative intensities are not normalized). ( $\longrightarrow$ ) Smectic phase at $T=318 \mathrm{~K},(--)$ Metastable form at $T=295 \mathrm{~K},(-\longrightarrow)$ Stable form at $T=300 \mathrm{~K}$.]

\section{Bibliographie}

[1] LaRsson, K. et Rand, R. P., Biochim. Biophys. Acta 326 (2) (1973) 245.

[2] Petrtcolas, W. L. et LipPert, J. L., Biochim. Biophys. Acta 282 (1972) 8.

[3] Wallach, D. F. H., Verma, S. P. et Fookson, J., Biochim. Biophys. Acta 559 (1979) 153.

[4] Casal, H. L., Cameron, D. G., Jarrell, H. C., Smith, I. C. P., Mantsch, H. H., Chem. Phys. Lipids 30 (1982) 17.

[5] Gaber, B. P. et Petitcolas, W. L., Biochim. Biophys. Acta 465 (1977) 260.

[6] SNyder, R. G., Scherer, J. R. et Gaber, B. P., Biochim. Biophys. Acta 601 (1980) 47.

[7] Snyder, R. G., Hsu, S. L., Krimm, S., Spectrochim. Acta A 34 (1978) 395.

[8] SNYder, R. G., SCHERER, J. R., J. Chem. Phys. 71 (8) (1979) 3221.

[9] Wunder, S. L., Merajver, S. D., J. Chem. Phys. 74 (10) (1981) 5341.

[10] Abbate, S., Zerbi, G., Wunder, S. L., J. Phys. Chem. 86 (1982) 3140.

[11] MacPhail, R. A., Snyder, R. G., Strauss, H. L., J. Chem. Phys. 77 (3) (1982) 1118.

[12] Snyder, R. G., Strauss, H. L., Elliger, C. A., J. Phys. Chem. 86 (1982) 5145.

[13] MacPhail, R. A., Strauss, H. L., Snyder, R. G., Elliger, C. A., J. Phys. Chem. 88 (1984) 334.

[14] Lydon, J. E. et Coakley, C. J., J. Physique Colloq. 36 (1975) C1-45.

[15] Leadbetter, A. J., Frost, J. C., Gaughan, J. P., Gray, G. W., Mosley, A., J. Physique 40 (1979) 375.

[16] Levelut, A. M., Zaghloul, B., Hardouin, F., J. Physique Lett. 43 (1982) L-83.

[17] Guillon, D. et Skoulios, A., Mol. Cryst. Liq. Cryst. 91 (1983) 341.

[18] De Jeu, W. J., Solid State Commun. 41 (7) (1982) 529.

[19] Ogorodnik, K. Z., Acta Phys. Pol. A 55 (1979) 935.

[20] Cox, R. J. et Johnson, J. F., IBM J. Res. Dev. 22 (1978) 51.

[21] Gray, G. W., Harrisson, K. J., Nash, J. A., Constant, J., Hulme, D. S., Kirton, J., Raynes, E. P., dans : Ordered Fluids and Liquid Crystals, Ed. R. S. Porter and J. F. Johnson (Wiley Interscience Publishers, New York) 1974, p. 617.

[22] Guillon, D. et Skoulios, A., J. Physique 45 (1984) 607.

[23] Le Brumant, J., Jaffrain, M. et Lacrampe, G., J. Phys. Chem. 88 (1984) 1548.

[24] Haase, W., Paulus, H. et Pendzialek, R., Mol. Cryst. Liq. Cryst. 100 (1983) 211.

[25] Gray, G. W. et Mosley, A., Mol. Cryst. Liq. Cryst. 35 (1976) 71. 